\title{
Laparoscopic sleeve gastrectomy in patients aged 55 and older
}

\begin{abstract}
Background: Morbid obesity is on the rise worldwide leading to an increase in bariatric surgery in different age groups. The objective of this study was to determine the safety and feasibility of Laparoscopic Sleeve Gastrectomy (LSG) in patients aged 55 and older.

Methods: 1200 patients in Al Amiri Hospital, Kuwait underwent LSG between 2009 and 2012. Forty four patients were aged 55years and older. Retrospective analysis included length of stay, percentage of excessive weight loss (EWL\%), Body Mass Index (BMI), and post-operative complications. All data was analyzed using SPSS.

Results: Mean age was 58 years, $70.5 \%$ were females. Mean pre-operative weight of $122 \mathrm{~kg}$ and BMI of $47 \mathrm{~kg} / \mathrm{m}^{2}$ was noted. Median length of stay was three days. Patients were followed up from two weeks to two years. Mean EWL\% was of $56 \%$ and $58 \%$ at one year and 18 months, respectively. Mean BMI reduced significantly over different time periods. Postoperative complications involved leak (2.3\%), biliary colic $(4.5 \%)$, renal embolism $(2.3 \%)$. Both age groups achieved successful weight loss; however, those below age 55 achieved significantly better results ( $\mathrm{p}$-value $<0.05$ ).
\end{abstract}

Conclusion: LSG has proven to be effective and safe in patients age 55 and older. The outcomes of patients aged 55 and older who undergo LSG should be further investigated among a larger sample size.

Keywords: Kuwait, sleeve, obesity, elderly
Volume 4 Issue I - 2016

\author{
Salman Al Sabah, ${ }^{2}$ Nourah Alsharqawi,' \\ Ahmed Al-Mulla, ${ }^{2}$ Shehab Ekrouf, ${ }^{3}$ Saud Al \\ Subaie, ${ }^{3}$ Muhannad Al Haddad, ${ }^{3}$ Khaled Al \\ Enezi, ${ }^{2}$ Talib Jumaa ${ }^{2}$ \\ 'Assistant Registrar, Department of Surgery, Al Amiri Hospital, \\ Kuwait \\ ${ }^{2}$ Consultant, Department of Surgery, Al Amiri Hospital, Kuwait \\ ${ }^{3}$ Specialist, Department of Surgery, Al Amiri Hospital, Kuwait
}

Correspondence: Salman Al Sabah, Consultant, AI Amiri Hospital, Kuwait City, Kuwait, Tel +965 666669II, Fax +96522464727, Email salman.k.alsabah@gmail.com

Received: March 27, 2015 | Published: January 29, 2016
Abbreviations: LSG, laparoscopic sleeve gastrectomy; EWL, excessive weight loss; BMI, body mass index; WHO, world health organization

\section{Introduction}

During the past century, a gradual increase of obesity has been noted. Malnutrition holds the attention of ministries and the media. Over the last two decades, emerging non-communicable diseases peaked in the 1950 s due to changes in diet and lifestyle, which have been increasing in developing countries, leading to an increased burden of disease. ${ }^{1}$ This has led to an increase in the requirement of health services, hence negatively impacting the country's development. The World Health Organization has reported that nearly 36 million disability adjusted life years are attributed to overweight or obesity and nearly 3 million people die eachyear. ${ }^{2}$

According to the WHO, most of the world's population being overweight and obese cause's death more than being underweight and approximately 1 billion people are overweight and 300million people are obese. ${ }^{3}$ In Kuwait, $33.8 \%$ and $40.8 \%$ of the Kuwaiti male and female population, respectively, were recorded to be obese in 2009 due to food habits and lifestyle choices that proved to be harmful to the populations' health. ${ }^{4}$ Owing to this continuous rise in obesity in Kuwait, bariatric surgery has also been on the rise. The prevalence of diabetes, ischemic heart disease and hypertension is gradually rising in Kuwait. The prevalence of Metabolic Syndrome (National Cholesterol Education Program Adult Treatment Panel III) was 37.7\% in females and $34.2 \%$ in males aged 20 and above. ${ }^{5}$

Bariatric surgery has been approved as reasonable management of elderly patients that fulfill the nationally established criteria for weight loss surgery. ${ }^{6}$ However, limited data with regards to the outcomes of bariatric surgery in elderly patients, success rates, peri-operative and post-operative morbidity and mortality has been documented. The main objective of this study is to determine the weight loss, post-operative complications, excessive weight loss percentage and morbidity and mortality in patients aged 55 and older, which underwent Laparoscopic Sleeve Gastrectomy (LSG).

\section{Methods}

A retrospective analysis of 1200 patients underwent LSG, of which 44 patients were aged 55 and above from February 2009 to October 2012 in Al Amiri Hospital database, Kuwait. Approval was obtained from Al Amiri Hospital to gain access to all patients' files. Patients' age, gender, nationality, pre-operative and post-operative co-morbidities and daily medications were gathered and deemed relevant. In addition, surgical data collected involved length of stay in the hospital and post-operative complications. Weight, Body Mass Index (BMI), and Percent of Excessive Weight loss (EWL\%) were collected for all patients pre-operatively and post-operatively during outpatient follow-ups over a period of 2 weeks to 2 years. A number of five patients that underwent previous bariatric surgery were excluded.

All procedures were performed by board certified surgeons in Al Amiri Hospital, Kuwait. The procedures were performed laparoscopically. A five port technique was used, utilizing a $36 \mathrm{~F}$ calibration tube and resecting four to six centimeters away from the pylorus. All patients that underwent LSG were qualified for bariatric surgery by utilizing the 1991 National Institutes of Health criteria. ${ }^{7}$ All patients under went pre-operative evaluation. A multi-disciplinary approach was applied by enlisting the consultation of dieticians and internal medicine specialties. Furthermore, laboratory investigations were performed to assess the patients' tolerance of the procedure and possible outcomes. Tests performed were a complete blood count, 
electrolytes, renal and liver function tests, blood sugar and hemoglobin $\mathrm{A} 1 \mathrm{C}$ and vitamin levels. In addition, all patients underwent a preoperative endoscopy ${ }^{8}$ and ultrasound of the abdomen.

Length of stay in the hospital was recorded in days from the day of admission to the day of discharge. Post-operative complications were documented, in addition to ICU admissions. Patients' pre-operative comorbidities were noted with daily medications use. Changes in preoperative comorbidities were evaluated and highlighted by performing relevant laboratory investigations during each follow up visit. All data was collected retrospectively by scheduling follow up visits at two weeks, three months, six months, 12 months and 18 months with periodical weight measurements.

\section{Analysis of data}

Statistical data analysis was performed by using SPSS version 18. Data was conveyed as mean or median depending on the criteria fulfilled. Quantitative data was compared by utilizing a paired $t$ test. Comparison between age groups was assessed via an independent $\mathrm{t}$ test. Results expressing a $\mathrm{p}$ value less than 0.05 were deemed statistically significant.

\section{Results}

A total of 44 patients fulfilled the inclusion criteria for data analysis. The mean patient age was 58.32years old (mean $\pm \mathrm{SD}$ ), of which 31 were female patients $(70.5 \%)$. A mean pre-operative weight of $121.80 \pm 21.35 \mathrm{~kg}$ was noted with a mean BMI of $46.95 \pm 6.01 \mathrm{~kg} / \mathrm{m}^{2}$. Patients were followed up starting from a minimum of 2 weeks up to a maximum of 2 years (median was 3 months). Eight of these 44 patients $(18.2 \%)$ failed to follow up in the outpatient clinic.

Patients presented with a substantial number of comorbidities preoperatively. These comorbidities had been previously detected and managed their primary care physicians and specialists. The top three comorbidities present were hypertension, diabetes mellitus and hypercholesteremia, in that order (Table 1). 16 patients were diagnosed diabetics, of which 9 patients diabetes had resolved (discontinue all diabetes-related medications and maintain a glycosylated hemoglobin less than 6.5\%) after the procedure. 12 patients suffered from hypercholesteremia, 2 patients condition had resolved (medications were discontinued and cholesterol levels were maintained within normal limits). 17 patients were known to be hypertensive, of which 4 patients now have a normal controlled blood pressure off medications. There was a zero percent conversion rate. The median length of stay recorded was 3days (1day - 90days).

Average weight and BMI were significantly reduced during all follow up periods. An average EWL\% of $52.5 \%$ after follow up for one year was demonstrated. 17 patients were followed up to one year, 11 of which managed to achieve EWL \% greater than 50\%. The

Table 3 Follow up intervals and change in EWL\%, BMI and Weight greatest EWL\% achieved was at 18 months, whence a mean score of $58.15 \%$ was documented.

Table I Pre-operative comorbidities

\begin{tabular}{lll}
\hline Comorbidity & Patients (n) & Resolved cases (n) \\
\hline Hypertension & 17 & 4 \\
Hypercholesterolemia & 12 & 2 \\
Ischemic Heart Disease & $\mathrm{I}$ & 0 \\
Diabetes & 16 & 9 \\
Lower Extremity Edema & $\mathrm{I}$ & $\mathrm{I}$ \\
Obstructive Sleep Apnea & $\mathrm{I}$ & $\mathrm{I}$ \\
Osteoarthritis & 2 & 0 \\
Gastritis & $\mathrm{I}$ & 0 \\
\hline
\end{tabular}

A total of 4 patients experienced complications. Complications experienced include biliary colic, myocardial infarction, staple line leak and renal embolism. One patient developed a gastric line leak $(2.3 \%)$ and required admission to the Intensive Care Unit for a period of 90days. This patient later underwent Roux-en-Y Gastric Bypass (RYGB) procedure as a treatment modality for the staple line leak. Two patients developed biliary colic (4.5\%) and underwent laparoscopic cholecystectomy and one patient developed renal embolism (2.3\%). No mortality was documented (Table 2).

Table 2 Post-operative complications

\begin{tabular}{lll}
\hline Complication & Patients (n) & Percentage (\%) \\
\hline Death & 0 & 0 \\
Gastric Leak & I & 2.3 \\
Biliary Colic & 2 & 4.5 \\
Myocardial Infarction & I & 2.3 \\
Renal Embolism & I & 2.3
\end{tabular}

Due to the controversy related to the effectiveness of LSG in elderly patients, data regarding EWL\% in different age groups were compared. In this study, patients were divided into two age groups: less than age 55 and age older than 55 . However, with data retrieved from the master database of 1200 patients, of all ages, that underwent LSG in Al Amiri Hospital, a significant EWL\% was detected after 1year. Although, a significant difference with a p-value of 0.018 in EWL\% was detected between the age groups less than 55years old and greater than 55years old, the former being higher, both groups still achieved an EWL\% of more than $50 \%$ at one year after the procedure (Table 3).

\begin{tabular}{llllll}
\hline & Pre-Operative & $\mathbf{3}$ months $(\mathbf{n}=\mathbf{3 2})$ & $\mathbf{6}$ months $(\mathbf{n}=\mathbf{2 7})$ & $\mathbf{1 2}$ months $(\mathbf{n}=\mathbf{1 7})$ & $\mathbf{1 8}$ months $(\mathbf{n}=\mathbf{1 0})$ \\
\hline Weight $(\mathrm{kg})$ & 122 & 105 & 98.7 & 95.8 & 92.1 \\
BMI' $\left(\mathrm{kg} / \mathrm{m}^{2}\right)$ & 47 & 40.3 & 37.8 & 35.5 & 34.9 \\
EWL\% & - & 32.2 & 44.9 & 55.8 & 58.1 \\
\hline
\end{tabular}

'Body Mass Index

${ }^{2}$ Percentage of Excessive Weight Loss 


\section{Discussion}

Bariatric surgery has been proved to be safe and effective for the treatment of obesity in the elderly. ${ }^{9}$ Many studies have proved that bariatric surgeries performed in high volume centers by experienced surgeons with appropriate post-operative care are associated with low morbidity and mortality rates. ${ }^{10}$ Although insufficient data is present in literature regarding the outcomes of LSG in the elderly population, mortality rates of $0.7 \%$ were recorded in well endorsed centers in those patients aged 60 and above. ${ }^{11}$ Although the frequency of postoperative morbidities has been documented to be low, LSG remains to be performed at low rates regardless of the increasing prevalence of obesity in the elderly population.

Over the years there has been a long ongoing argument about the exact definition of "elderly". A range of 50 to 65 has been defined by many institutions leading to a debate regarding the definition of elderly. Recently, the United Nations established a cut-off age of above 60years old. ${ }^{12}$ However, in our study, a range of 55 and above was used as 9 patients above 60years old underwent LSG.

From the few articles published that address the outcomes of bariatric surgery in the elderly population, many have demonstrated an increase in peri-operative mortality and morbidity. A recent study carried out, confirmed that only $21 \%$ of bariatric centers perform more than 4 procedures peryear. ${ }^{9}$ Livingston and Langert stated an adverse event rate of more than $20 \%$ with a mortality rate of $3.2 \%$; younger individuals, in comparison to elderly patients achieved better weight loss outcomes as well as comorbidity control, which is consistent with our study. ${ }^{13}$

This study proves that elderly patients may experience a successful weight loss procedure with low mortality rate and appropriate morbidity rates. This is in comparison to other literatures that have confirmed the same results, whereby all patients in the study had a 30day mortality rate of $0 \% .{ }^{9}$ The Longitudinal Assessment of Bariatric Surgery Consortium stated a nationally accepted 30day mortality rate of $0.3 \% .{ }^{14} \mathrm{In}$ addition, the morbidity occurrences in our study are deemed acceptable in comparison to other studies. A range of $0.7 \%$ to $5 \%$ (mean is $2.3 \%$ ) has been established for gastric leak by collecting the results of many studies. ${ }^{15}$ Our result, $2.3 \%$, is consistent with another study and is within the acceptable range. ${ }^{16}$ Furthermore, our patients had minimal serious complications, whereby only one patient $(2.3 \%)$ was admitted to the Intensive Care Unit and one patient $(2.3 \%)$ developed myocardial infarction a year after the procedure. BMI significantly reduced with each time period (2weeks, 3months, 6 months, 1year and 18months) for all patients. Lastly, our patients demonstrated an improvement in their well-being and reduction of daily use of medications.

\section{Conclusion}

This study illustrates that LSG is safe and effective in patients aged 55 and above. Although those below 55 achieved better EWL\%, the mean EWL\% at 1year was more than $50 \%$ for patients aged 55 and above. All our patients demonstrated a significant weight loss and decline in BMI over different time periods. Significant resolution of comorbidities was also noted. However, despite these significant results, larger and longer follow-up studies are required for validation and verification of these results.

\section{Acknowledgements}

None.

\section{Conflict of interest}

The author declares no conflict of interest.

\section{References}

1. Amuna P, Zotor FB. Epidemiological and nutrition transition in developing countries: impact on human health and development. Proc Nutr Soc. 2008;67(1):82-90.

2. WHO. Obesity. World Health Organization; 2013.

3. WHO. Global health risks: mortality and burden of disease attributable to selected major risks. Switzerland: World Health Organization; 2009.

4. Ministry of Health. Annual reports-Kuwait surveillance system, 20012009. Kuwait: Ministry of Health; 2010.

5. Al Zenki S, Al Omirah H, Al Hooti S, et al. High prevalence of metabolic syndrome among Kuwaiti adults-a wake-up call for public health intervention. Int J Environ Res Public Health. 2012;9(5):1984-1996.

6. Villareal DT, Apovian CM, Kushner RF. Obesity in older adults: technical review and position statement of the American Society for Nutrition and NAASO, The Obesity Society 1,2,3,4,5. The American Journal for Clinical Nutrition. 2005;82(5):923-934.

7. National Institutes of Health. Clinical guidelines on the identification, evaluation, and treatment of overweight and obesity in adults: the evidence report. Obes Res. 1998;6:51S-209S.

8. Almazeedi S, Al-Sabah S, Al-Mulla A, et al. Gastric Histopathologies in Patients Undergoing Laparoscopic Sleeve Gastrectomies. Obes Surg. 2013;23(3):314-319.

9. O’Keefe KL, Kemmeter PR, Kemmeter KD. Bariatric Surgery Outcomes in Patients Aged 65Years and Older at an American Society for Metabolic and Bariatric Surgery Center of Excellence. Obes Surg. 2010;20(9):1199-1205.

10. Nguyen NT, Paya M, Stevens M, et al. The relationship between hospital volume and outcome in bariatric surgery at academic medical centers. Ann Surg. 2004;240(4):586-594.

11. Fatima J, Houghton SG, Iqbal CW, et al. Bariatric Surgery at the Extremes of Age. J Gastrointest Surg. 2006;10(10):1392-1396.

12. WHO. Definition of an older or elderly person: Proposed Working Definition of an Older Person in Africa for the MDS Project. World Health Organization; 2013.

13. Livingston EH, Langert J. The Impact of Age and Medicare Status on Bariatric Surgical Outcomes. Arch Surg. 2006;141(11):1115-1120.

14. Flum DR. Longitudinal Assessment of Bariatric Surgery (LABS) Consortium. Perioperative safety in the longitudinal assessment of bariatric surgery. N Engl J Med. 2009;361(5):445-454.

15. Márquez MF, Ayza MF, Lozano RB, et al. Gastric Leak after Laparoscopic-Sleeve Gastrectomy for Obesity. Obesity Surgery. 2010;20(9):1306-1311.

16. Felberbauer FX, Langer F, Shakeri-Manesch S, et al. Laparoscopic sleeve gastrectomy as an isolated bariatric procedure: intermediateterm results from a lage series in three Austrian centers. Obes Surg. 2008;18(7):814-818 